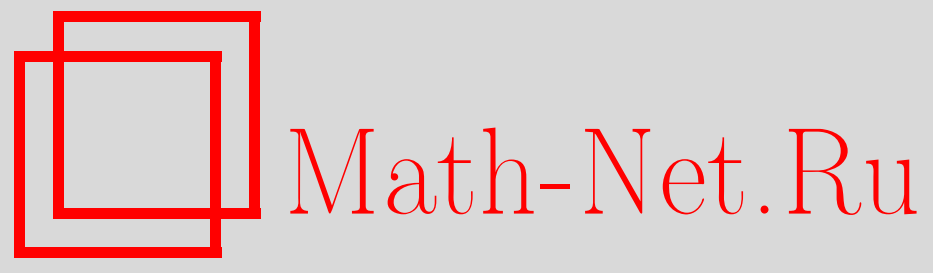

И. В. Кудинов, О. Ю. Курганова, В. К. Ткачев, Получение точного аналитического решения стационарной двумерной задачи теплопроводности с источником теплоты, Вестн. Сам. гос. техн. ун-та. Сер. Физ.-мат. науки, 2019, номер 1, 195-203

DOI: https://doi.org/10.14498/vsgtu1665

Использование Общероссийского математического портала MathNet.Ru подразумевает, что вы прочитали и согласны с пользовательским соглашением

http://www.mathnet.ru/rus/agreement

Параметры загрузки:

IP : 35.173 .137 .237

26 апреля 2023 г., $10: 16: 42$

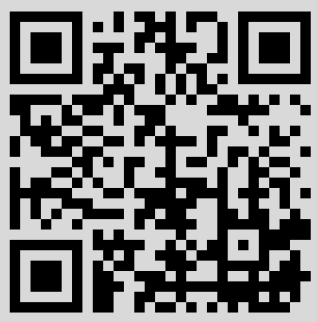


Вестн. Сам. гос. техн. ун-та. Сер. Физ.-мат. науки. 2019. Т. 23, № 1. С. $195-203$ ISSN: 2310-7081 (online), 1991-8615 (print)

УДК 517.958:[536.2+539.219.3]

\title{
Получение точного аналитического решения стационарной двумерной задачи теплопроводности с источником теплоты
}

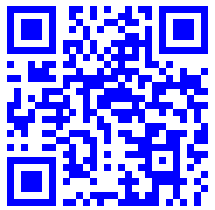

\author{
И. В. Кудинов, О. Ю. Курганова, В. К. Ткачев \\ Самарский государственный технический университет, \\ Россия, 443100, Самара, ул. Молодогвардейская, 244.
}

\section{Аннотация}

На основе ортогонального метода Бубнова-Галеркина с использованием тригонометрических систем координатных функций получено точное аналитическое решение стационарной двумерной задачи теплопроводности для бесконечно-протяженного бруса квадратного сечения с источником теплоты. Благодаря свойству ортогональности тригонометрических координатных функций получаемая в методе Бубнова-Галеркина бесконечная система обыкновенных дифференциальных уравнений разделяется и приводится к решению одного обобщенного уравнения, что позволяет получить точное аналитическое решение простого вида в виде бесконечного ряда. В силу симметричности задачи рассматривается лишь четверть поперечного сечения бруса при задании по линиям разреза граничных условий адиабатной стенки (отсутствия теплообмена), что позволяет (в отличие от известного классического точного аналитического решения) значительно упростить как процесс получения решения, так и окончательное выражение для него.

Ключевые слова: уравнение Пуассона, двумерная краевая задача, источник теплоты, метод Бубнова-Галеркина, ортогональная система координатных функций, точное аналитическое решение.

Получение: 14 декабря 2018 г. / Исправление: 19 января 2019 г. Принятие: 27 января 2019 г. / Публикация онлайн: 13 февраля 2019 г.

\section{Краткое сообщение}

(웅 Контент публикуется на условиях лицензии Creative Commons Attribution 4.0 International (https://creativecommons.org/licenses/by/4.0/deed.ru)

\section{Образец для цитирования}

Кудинов И. В., Курганова О. Ю., Ткачев В. К. Получение точного аналитического решения стационарной двумерной задачи теплопроводности с источником теплоты // Вестн. Сам. гос. техн. ун-та. Сер. Физ.-мат. науки, 2019. Т. 23, № 1. С. 195-203. doi: 10.14498/vsgtu1665.

\section{Сведения об авторах}

Игорь Васильевич Кудинов (1) https://orcid.org/0000-0002-9422-0367

доктор технических наук; доцент; каф. теоретических основ теплотехники и гидромеханики; e-mail: igor-kudinov@bk.ru

Ольга Юръевна Курганова (1) https://orcid.org/0000-0003-4767-2544 аспирант; каф. теоретических основ теплотехники и гидромеханики; e-mail: o.y.kurganova@gmail.com

Василий Константинович Ткачев (1) https://orcid.org/0000-0002-0375-2369 аспирант; каф. теоретических основ теплотехники и гидромеханики;

e-mail: tvk93@yandex.ru 
Получение аналитических решений двумерных краевых задач на основе уравнения Пуассона представляет значительный практический интерес изза их широкого применения при анализе различных физических процессов (теплопроводности, течения жидкостей, теории упругости, термоупругости, кручения призматических тел и др.) [1-5]. Трудность их решения объясняется двумерностью краевой задачи и неоднородностью исходного дифференциального уравнения. Известное точное аналитическое решение представляет сложный бесконечный функциональный ряд, содержащий гиперболические функции [5].

Из приближенных аналитических методов решения краевых задач теплопроводности большое распространение получил интегральный метод теплового баланса, относящийся к группе ортогональных методов взвешенных невязок $[6-14,19]$. С его помощью можно получать приближенные аналитические решения краевых задач, получение точных решений которых не предоставляется возможным (нелинейные краевые задачи с переменными физическими свойствами среды и др.). Однако основным недостатком этих методов является низкая точность, объясняемая тем, что при их использовании требуется выполнение осредненного исходного уравнения (интеграла теплового баланса). Применение дополнительной искомой функции и дополнительных граничных условий позволяет удовлетворить исходное дифференциальное уравнение в зависимости от числа приближений искомого решения с заданной степенью точности. Отметим, что методы, основанные на выполнении уравнения в граничных точках, рассмотрены также в работах [15-18].

В настоящей работе точное аналитическое решение получено на основе метода Бубнова-Галеркина с использованием ортогональных систем тригонометрических координатных функций, что позволяет существенно упростить как процесс получения решения, так и окончательное выражение для него из-за возможности сведения решения бесконечной системы обыкновенных дифференциальных уравнений к интегрированию одного обобщенного дифференциального уравнения. Основную идею метода рассмотрим на примере решения задачи теплопроводности для бесконечного бруса квадратного сечения с источником теплоты в следующей математической постановке:

$$
\begin{gathered}
\frac{\partial^{2} T(x, y)}{\partial x^{2}}+\frac{\partial^{2} T(x, y)}{\partial y^{2}}=-\frac{\nu}{\lambda}, \quad 0<x<\delta, 0<y<\delta \\
T(\delta, y)=T_{b},\left.\quad \frac{\partial T(x, y)}{\partial x}\right|_{x=0}=0,\left.\quad \frac{\partial T(x, y)}{\partial y}\right|_{y=0}=0, \quad T(x, \delta)=T_{b},
\end{gathered}
$$

где $T$ - температура, $\mathrm{K} ; x, y$ - координаты, м; $\nu$ - мощность внутреннего источника теплоты, Вт $/ \mathrm{m}^{3} ; \lambda$ - коэффициент теплопроводности, Вт $/(\mathrm{M} \cdot \mathrm{K}) ; T_{b}-$ температура стенки, K; $\delta$ - половина стороны сечения бруса, м.

Ввиду симметрии температурного поля рассматривается только четверть поперечного сечения бруса. В работе [7] на основе определения дополнительной искомой функции и дополнительных граничных условий получено приближенное аналитическое решение задачи (1), (2) (второе приближение). Однако нахождение решения для большего числа приближений затруднительно в виду громоздкости получаемых выражений, что связано с использованием сложного вида дополнительных граничных условий. С целью упрощения процесса получения точного аналитического решения введём следующие без- 
размерные переменные и параметры:

$$
\Theta=\frac{T-T_{b}}{T_{b}}, \quad \xi=\frac{x}{\delta}, \quad \eta=\frac{y}{\delta}, \quad B=\frac{\nu \delta^{2}}{\lambda T_{b}},
$$

где $\Theta$ - безразмерная температура; $\xi, \eta$ - безразмерные координаты $(\xi \in[0,1]$, $\eta \in[0,1]) ; B$ - безразмерный параметр.

С учетом обозначений (3) задача (1), (2) запишется следующим образом:

$$
\begin{gathered}
\frac{\partial^{2} \Theta(\xi, \eta)}{\partial \xi^{2}}+\frac{\partial^{2} \Theta(\xi, \eta)}{\partial \eta^{2}}+B=0, \quad 0<\xi<1,0<\eta<1 ; \\
\left.\frac{\partial \Theta(\xi, \eta)}{\partial \xi}\right|_{\xi=0}=0 \\
\Theta(1, \eta)=0 \\
\left.\frac{\partial \Theta(\xi, \eta)}{\partial \eta}\right|_{\eta=0}=0 \\
\Theta(\xi, 1)=0 .
\end{gathered}
$$

Решение задачи (4)-(8) принимается в виде

$$
\Theta(\xi, \eta)=\sum_{k=1}^{\infty} q_{k}(\xi) \varphi_{k}(\eta)
$$

где $q_{k}(\xi)$ - неизвестные коэффициенты; $\varphi_{k}=\cos \left(\frac{2 k-1}{2} \pi \eta\right)-$ координатные функции, $k=1,2, \ldots$

Соотношение (9) благодаря принятой системе координатных функций точно удовлетворяет граничным условиям (7), (8). Для определения неизвестных функций $q_{k}(\xi)$, следуя методу Бубнова-Галеркина, составим невязку уравнения (4) и потребует ее ортогональности ко всем координатным функциям $\varphi_{j}=\cos \left(\frac{2 j-1}{2} \pi \eta\right)$ :

$$
\int_{0}^{1}\left[\frac{\partial^{2} \Theta(\xi, \eta)}{\partial \xi^{2}}+\frac{\partial^{2} \Theta(\xi, \eta)}{\partial \eta^{2}}+B\right] \varphi_{j}(\eta) d \eta=0, \quad j=1,2, \ldots
$$

Подставляя (9) в (10), находим

$$
\begin{aligned}
\int_{0}^{1} \sum_{k=1}^{\infty}\left[q_{k}^{\prime \prime}(\xi) \varphi_{k}(\eta)-\left(\frac{2 k-1}{2}\right)^{2} \pi^{2} q_{k}(\xi) \varphi_{k}(\eta)\right] \varphi_{j}(\eta) d \eta+ & \\
& +B \int_{0}^{1} \varphi_{j}(\eta) d \eta=0, \quad j=1,2, \ldots
\end{aligned}
$$

Соотношение (11) представляет бесконечную систему обыкновенных дифференциальных уравнений второго порядка относительно неизвестных функций $q_{k}(\xi)$. Но из-за ортогональности введенных тригонометрических функций (11) распадается на следующие дифференциальные уравнения:

$$
q_{k}^{\prime \prime}(\xi)-\left(\frac{2 k-1}{2}\right)^{2} \pi^{2} q_{k}(\xi)+\frac{(-1)^{k+1} 4 B}{(2 k-1) \pi}=0, \quad k=1,2, \ldots
$$


Интегрируя уравнение (12), находим

$$
q_{k}(\xi)=C_{1 k} \exp \left(-\frac{2 k-1}{2} \pi \xi\right)+C_{2 k} \exp \left(\frac{2 k-1}{2} \pi \xi\right)+\frac{(-1)^{k+1} 16 B}{(2 k-1)^{3} \pi^{3}}
$$

где $C_{1 k}, C_{2 k}$ - постоянные интегрирования, $k=1,2, \ldots$

Подставляя (13) в (9), получаем

$$
\begin{array}{r}
\Theta(\xi, \eta)=\sum_{k=1}^{\infty}\left[C_{1 k} \exp \left(-\frac{2 k-1}{2} \pi \xi\right)+C_{2 k} \exp \left(\frac{2 k-1}{2} \pi \xi\right)+\right. \\
\left.+\frac{(-1)^{k+1} 16 B}{(2 k-1)^{3} \pi^{3}}\right] \varphi_{k}(\eta) .
\end{array}
$$

Для определения постоянных интегрирования $C_{1 k}$ и $C_{2 k}$ составим невязку граничных условий (5), (6) и потребуем ортогональности невязки ко всем координатным функциям $\varphi_{j}(\eta)$ :

$$
\left.\int_{0}^{1} \frac{\partial \Theta(\xi, \eta)}{\partial \xi}\right|_{\xi=0} \varphi_{j}(\eta) d \eta=0, \quad \int_{0}^{1} \Theta(1, \eta) \varphi_{j}(\eta) d \eta=0, \quad j=1,2, \ldots
$$

После подстановки (14) в (15) получим систему относительно неизвестных $C_{1 k}$ и $C_{2 k}$ :

$$
\left\{\begin{array}{l}
C_{2 k}-C_{1 k}=0 ; \\
C_{1 k} \exp \left(-\frac{2 k-1}{2} \pi\right)+C_{2 k} \exp \left(\frac{2 k-1}{2} \pi\right)+\frac{16 B(-1)^{k-1}}{(2 k-1)^{3} \pi^{3}}=0,
\end{array}\right.
$$

решение которой имеет вид

$$
C_{1 k}=C_{2 k}=\frac{(-1)^{k}}{\left(\frac{2 k-1}{2} \pi\right)^{3} \operatorname{ch}\left(\frac{2 k-1}{2} \pi\right)}
$$

Ряд (14) с учетом найденных значений (16) представляет собой точное аналитическое решение задачи (4)-(8). Анализ результатов расчетов позволяет заключить, что ряд (14) «быстро» сходится. Максимальная относительная погрешность в чебышевской норме от точного решения находится по формуле

$$
\Delta_{\max }=\left|\frac{\Theta(\xi, \eta)-\Theta_{\mathrm{ex}}(\xi, \eta)}{\Theta_{\mathrm{ex}}(\xi, \eta)}\right| \cdot 100 \%
$$

где $\Theta_{\mathrm{ex}}(\xi, \eta)$ - точное решение, представленное в [5]. При использовании одного члена ряда (14) (штриховые линии на рисунке) погрешность $\Delta_{\max }$ не превышает $6 \%$, при использовании двух членов - не более $1.5 \%$, а при использовании трех членов ряда (сплошные линии на рисунке) - менее $1 \%$. Точное решение на рисунке не приведено, поскольку визуально оно неотличимо от решения при трех членах. 


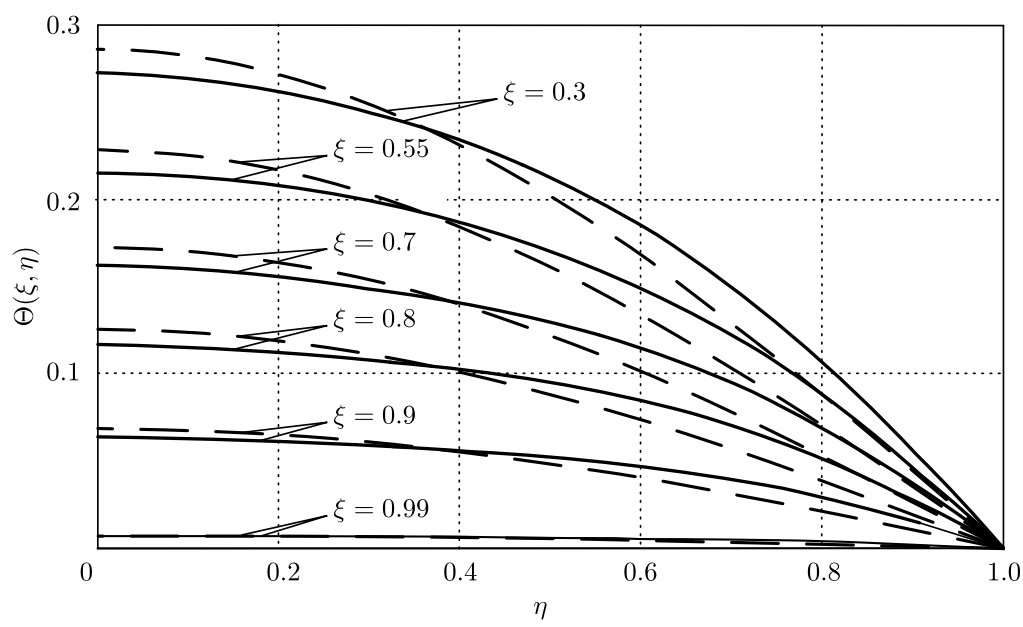

Распределение температуры в различных сечениях бруса. Расчеты по формуле (14): штриховые линии $-n=1$, сплошные линии $-n=3$, где $n$ - число членов ряда (14), $B=1$

[Temperature distribution in different cross-sections of the bar. Calculations are made by Eq. (14): dashed lines for $n=1$, solid lines for $n=3$, where $n$ is the number of terms in the series $(14), B=1]$

Отметим, что полученные по формуле (14) результаты в различных точках сечения бруса при любом количестве членов ряда (14) численно полностью совпадают с решением, приведенным в [5], однако отличаются простотой получения с безразмерным диапазоном изменения пространственных переменных $(\xi \in[0,1], \eta \in[0,1])$ и возможностью изменения величины внутреннего источника теплоты $B$.

\section{Выводы}

1. На основе ортогонального метода Бубнова-Галеркина при использовании тригонометрических координатных функций получено точное аналитическое решение стационарной двумерной задачи теплопроводности для бесконечно-протяженного бруса квадратного сечения с объемным источником теплоты.

2. Благодаря ортогональности тригонометрических координатных функций, неизвестные функции $q_{k}(\xi)$ в бесконечной системе обыкновенных дифференциальных уравнений, получаемых в результате применения метода Бубнова-Галеркина, расщепляются, что позволяет получить решение, точно удовлетворяющее дифференциальному уравнению и всем граничным условиям краевой задачи.

Конкурирующие интересы. У нас нет конкурирующих интересов.

Авторская ответственность. Мы несем полную ответственность за предоставление окончательной версии рукописи в печать. Окончательная версия рукописи нами одобрена.

Финансирование. Работа выполнена при поддержке Российского фонда фундаментальных исследований (проект № 18-38-00029 мол_а).

\section{Библиографический список}

1. Chen C. S., Muleshkov A. S., Golberg M. A., Mattheij R.M.M. A mesh-free approach to 
solving the axisymmetric Poisson's equation// Numer. Meth. Part. D. E., 2005. vol.21, no. 2. pp. 349-367. doi: 10.1002/num. 20040.

2. Тихонов А. Н., Самарский А. А. Уравнения математической физики. М.: Физматлит, 1999. $798 \mathrm{c}$.

3. Глазунов Ю. Т. Вариационные методы. М.-Ижевск: Регулярная и хаотическая динамика; Институт компьютерных исследований, 2006. 470 с.

4. Цой П. В. Системные методы расчета краевых задач тепломассопереноса. М.: МЭИ, 2005. 568 c.

5. Канторович Л. В., Крылов В. И. Приближенные методы высшего анализа. Л.: Физматгиз, 1950. 695 с.

6. Кудинов В. А., Кудинов И. В. Об одном методе получения точного аналитического решения гиперболического уравнения теплопроводности на основе использования ортогональных методов // Вестн. Сам. гос. техн. ун-та. Сер. Физ.-мат. науки, 2010. № 5(21). C. 159-169. doi : 10.14498/vsgtu804.

7. Кудинов В. А., Кудинов И. В., Скворцова М. П. Обобщенные функции и дополнительные граничные условия в задачах теплопроводности для многослойных тел// Ж. вычисл. матем. и матем. физ., 2015. Т. 55, №4. С. 669-680. doi: 10.7868/ S0044466915040080.

8. Bollati J., Semitiel J., Tarzia D. A. Heat balance integral methods applied to the one-phase Stefan problem with a convective boundary condition at the fixed face // Appl. Math. Comp., 2018. vol. 331. pp. 1-19. doi: 10.1016/j.amc.2018.02.054.

9. Hristov J. The heat radiation diffusion equation explicit analytical solutions by improved integral-balance method // Thermal science, 2018. vol. 22, no. 2. pp. 777-788. doi : 10.2298/ TSCI171011308H.

10. Hristov J. Double integral-balance method the fractional subdiffusion equation: approximate solutions, optimization problems to be resolved and numerical simulations // J. Vib. Control, 2017. vol. 23, no. 17. pp. 2795-2818. doi: 10.1177/1077546315622773.

11. Hristov J. Multiple integral-balance method basic idea and an example with Mullin's model of thermal grooving // Thermal science, 2017. vol.21, no.3. pp. 1555-1560. doi: 10.2298/ TSCI170410124H.

12. Кудинов В. А., Стефанюк Е. В. Аналитический метод решения задач теплопроводности на основе введения фронта температурного возмущения и дополнительных граничных условий // Инженерно-физический журнал, 2009. Т. 82, № 3. С. 540-558.

13. Стефанюк Е. В., Кудинов В. А. Получение приближенных аналитических решений при рассогласовании начальных и граничных условий в задачах теории теплопроводности // Изв. вузов. Матем., 2010. № 4. С. 63-71.

14. Кудинов В. А., Дикоп В. В., Габдушев Р. Ж., Стефанюк С. А. Об одном методе определения собственных чисел в нестационарных задачах теплопроводности // Изв. РАН. Энергетика, 2002. № 4. С. 112-117.

15. Канторович Л. В. Об одном методе приближенного решения дифференциальных уравнений в частных производных // Докл. АН СССР, 1934. Т. 2, №9. С. 532-534.

16. Федоров Ф. М. Граничный метод решения прикладных задач математической физики. Новосибирск: Наука, 2000. 220 с.

17. Кудряшов Н. А. Приближенные решения одной задачи нелинейной теплопроводности // ЖК. вычисл. матем. и матем. физ., 2005. Т. 45, № 11. С. 2044-2051.

18. Wang G. T., Pei K., Agarwal R. P., Zhang L. H., Ahmad B. Nonlocal Hadamard fractional boundary value problem with Hadamard integral and discrete boundary conditions on a half-line// J. Comp. Appl. Math., 2018. vol.343. pp. 230-239. doi:10.1016/j.cam.2018. 04.062 .

19. Кудинов В. А., Клеблеев Р. М., Куклова Е. А. Получение точных аналитических решений нестационарных задач теплопроводности ортогональными методами // Becmн. Сам. гос. техн. ун-та. Сер. Физ.-мат. науки, 2017. Т. 21, №1. С. 197-206. doi: 10 . 14498/vsgtu1521. 
MSC: 35K05, 80A20, 35C10

\title{
Exact analytical solution for the stationary two-dimensional heat conduction problem with a heat source
}

\author{
I. V. Kudinov, O. Yu. Kurganova, V. K. Tkachev
}

Samara State Technical University,

244, Molodogvardeyskaya st., Samara, 443100, Russian Federation.

\begin{abstract}
The exact analytic solution for the stationary two-dimensional heat conduction problem with a heat source for an infinite square bar was obtained. It was based on the Bubnov-Galyorkin orthogonal method using trigonometric systems of coordinate functions. The infinite system of ordinary differential equations obtained by the Bubnov-Galyorkin method is divided and reduced by the orthogonality property of trigonometric coordinate functions to the solution of a generalized equation which provides the exact analytical solution in a simple form, i.e. in the form of an infinite series. In view of the symmetry of the problem, only a quarter of the cross-section of the bar is considered for the boundary conditions of the adiabatic wall (the absence of heat transfer) along the cut lines, which allows (in contrast to the well-known classical exact analytical solution) to significantly simplify the process of the solution and the final equation.
\end{abstract}

Keywords: Poisson equation, two-dimensional boundary value problem, heat source, Bubnov-Galyorkin method, orthogonal system of coordinate functions, exact analytic solution.

Received: $14^{\text {th }}$ December, $2018 /$ Revised: $19^{\text {th }}$ January, $2019 /$

Accepted: $27^{\text {th }}$ January, $2019 /$ First online: $13^{\text {th }}$ February, 2019

\section{Short Communication}

(2) (a) The content is published under the terms of the Creative Commons Attribution 4.0 International License (http://creativecommons.org/licenses/by/4.0/)

Please cite this article in press as:

Kudinov I. V., Kurganova O. Yu., Tkachev V. K. Exact analytical solution for the stationary two-dimensional heat conduction problem with a heat source, Vestn. Samar. Gos. Tekhn. Univ., Ser. Fiz.-Mat. Nauki [J. Samara State Tech. Univ., Ser. Phys. Math. Sci.], 2019, vol. 23, no. 1, pp. 195-203. doi: 10.14498/vsgtu1665 (In Russian).

\section{Authors' Details:}

Igor V. Kudinov (10) https://orcid.org/0000-0002-9422-0367

Dr. Techn. Sci.; Associate Professor; Dept. of Theoretical Foundations of Heat Engineering and Hydromechanics; e-mail: igor-kudinov@bk.ru

Olga Yu. Kurganova (D) https://orcid.org/0000-0000-4767-2544

Postgraduate Student; Dept. of Theoretical Foundations of Heat Engineering and Hydromechanics; e-mail: o.y.kurganova@gmail.com

Vasily K. Tkachev (1D https://orcid.org/0000-0002-0375-2369

Postgraduate Student; Dept. of Theoretical Foundations of Heat Engineering and Hydromechanics; e-mail: tvk93@yandex.ru 
Competing interests. We have no competing interests.

Author's Responsibilities. We take full responsibility for submitting the final manuscript in print. We approved the final version of the manuscript.

Financing. This work was supported by the Russian Foundation for Basic Research (project no. 18-38-00029 mol_a).

\section{References}

1. Chen C. S., Muleshkov A. S., Golberg M. A., Mattheij R.M.M. A mesh-free approach to solving the axisymmetric Poisson's equation, Numer. Meth. Part. D. E., 2005, vol. 21, no. 2, pp. 349-367. doi : 10.1002/num. 20040.

2. Tikhonov A. N., Samarskii A. A. Equations of mathematical physics, International Series of Monographs on Pure and Applied Mathematics, vol. 39. Oxford etc., Pergamon Press, 1963, $\mathrm{xvi}+765 \mathrm{pp}$.

3. Glazunov Yu. T. Variatsionnye metody [Variational Methods]. Moscow, Izhevsk, Regular and Chaotic Dynamics; Computer Research Institute, 2006, 470 pp. (In Russian)

4. Tsoi P. V. Sistemnye metody rascheta kraevykh zadach teplomassoperenosa [System Methods of Calculating the Boundary Value Problems of Heat and Mass Transfer]. Moscow, Moscow Energy Institute, 2005, 568 pp. (In Russian)

5. Kantorovich L. V., Krylov V. I. Approximate methods of higher analysis. Groningen, P. Noordhoff Ltd., 1958, xii+681 pp.

6. Kudinov V. A., Kudinov I. V. About one Method of Obtaining of the Exact Analytical Decision of the Hyperbolic Equation of Heat Conductivity on the Basis of Use of Orthogonal Methods, Vestn. Samar. Gos. Tekhn. Univ., Ser. Fiz.-Mat. Nauki [J. Samara State Tech. Univ., Ser. Phys. Math. Sci.], 2010, no.5(21), pp. 159-169 (In Russian). doi: 10.14498/ vsgtu804.

7. Kudinov V. A., Kudinov I. V., Skvortsova M. P. Generalized functions and additional boundary conditions in heat conduction problems for multilayered bodies, Comput. Math. Math. Phys., 2015, vol. 55, no. 4, pp. 666-667. doi : 10.1134/S0965542515040089.

8. Bollati J., Semitiel J., Tarzia D. A. Heat balance integral methods applied to the one-phase Stefan problem with a convective boundary condition at the fixed face, Appl. Math. Comp., 2018, vol.331, pp. 1-19. doi: 10.1016/j.amc.2018.02.054.

9. Hristov J. The heat radiation diffusion equation explicit analytical solutions by improved integral-balance method, Thermal science, 2018, vol.22, no. 2, pp. 777-788. doi: 10.2298/ TSCI171011308H.

10. Hristov J. Double integral-balance method the fractional subdiffusion equation: approximate solutions, optimization problems to be resolved and numerical simulations, J. Vib. Control, 2017, vol. 23, no. 17, pp. 2795-2818. doi : 10.1177/1077546315622773.

11. Hristov J. Multiple integral-balance method basic idea and an example with Mullin's model of thermal grooving, Thermal science, 2017, vol.21, no.3, pp. 1555-1560. doi: 10.2298/ TSCI170410124H.

12. Kudinov V. A., Stefanyuk E. V. Analytical solution method for heat conduction problems based on the introduction of the temperature perturbation front and additional boundary conditions, J. Eng. Phys. Thermophys., 2009, vol.82, no.3, pp. 537-555. doi:10.1007/ s10891-009-0223-8.

13. Stefanyuk E. V., Kudinov V. A. Approximate analytic solution of heat conduction problems with a mismatch between initial and boundary conditions, Russian Math. (Iz. VUZ), 2010, vol. 54, no. 4, pp. 55-61. doi : 10.3103/S1066369X10040079.

14. Kudinov V. A., Dikop V. V., Gabdushev R. Zh., Stefanyuk S. A. On a method for determining eigenvalues in nonstationary heat conduction problems, Izv. RAN. Energetika, 2002, no. 4, pp. 112-117 (In Russian).

15. Kantorovich L. V. On an approximation method for the solution of a partial differential equation, Dokl. Akad. Nauk SSSR, 1934, vol.2, no. 9, pp. 532-534 (In Russian). 
16. Fedorov F. M. Granichnyi metod resheniia prikladnykh zadach matematicheskoi fiziki [The boundary method for solving applied problems of mathematical physics]. Novosibirsk, Nauka, 2000, 220 pp. (In Russian)

17. Kudryashov N. A. Approximate solutions of nonlinear heat conduction problem, Comput. Math. Math. Phys., 2005, vol. 45, no. 11, pp. 1965-1972.

18. Wang G. T., Pei K., Agarwal R. P., Zhang L. H., Ahmad B. Nonlocal Hadamard fractional boundary value problem with Hadamard integral and discrete boundary conditions on a halfline, J. Comp. Appl. Math., 2018, vol.343, pp. 230-239. doi: 10.1016/j.cam.2018.04.062.

19. Kudinov V. A., Klebleev R. M., Kuklova E. A. Obtaining exact analytical solutions for nonstationary heat conduction problems using orthogonal methods, Vestn. Samar. Gos. Tekhn. Univ., Ser. Fiz.-Mat. Nauki [J. Samara State Tech. Univ., Ser. Phys. Math. Sci.], 2017, vol. 21, no. 1, pp. 197-206 (In Russian). doi: 10.14498/vsgtu1521. 